\title{
Explicit solutions for queues with Hypo-exponential service time and applications to product-form analysis
}

\author{
Andrea Marin \\ DAIS - Università Ca' Foscari Venezia \\ via Torino, 155 \\ Venice, Italy \\ marin@dsi.unive.it
}

\author{
Samuel Rota Bulò \\ DAIS - Università Ca' Foscari Venezia \\ via Torino, 155 \\ Venice, Italy \\ srotabul@dsi.unive.it
}

\begin{abstract}
Queueing systems with Poisson arrival processes and Hypoexponential service time distribution have been widely studied in literature. Their steady-state analysis relies on the observation that the infinitesimal generator matrix has a block-regular structure and, hence, matrix-analytic method may be applied. Let $\pi_{n k}$ be the steady-state probability of observing the $k$-th stage of service busy and $n$ customers in the queue, with $1 \leq k \leq K$, and $K$ the number of stages, and let $\boldsymbol{\pi}_{n}=\left(\pi_{n 1}, \ldots, \pi_{n K}\right)$. Then, it is well-known that there exists a rate matrix $\mathbf{R}$ such that $\boldsymbol{\pi}_{n+1}=\boldsymbol{\pi}_{n} \mathbf{R}$. In this paper we give a symbolic expression for such a matrix $\mathbf{R}$. Then, we exploit this result in order to address the problem of approximating a $M / \mathrm{HYPO}_{K} / 1$ queue by a model with initial perturbations which yields a product-form stationary distribution. We show that the result on the rate matrix allows us to specify the approximations for more general models than those that have been previously considered in literature and with higher accuracy.
\end{abstract}

\section{Categories and Subject Descriptors}

C.4 [PERFORMANCE OF SYSTEMS]: Modeling techniques

\section{General Terms \\ Performance}

\section{Keywords}

Queueing theory, Product-form solutions.

\section{INTRODUCTION}

The analysis of queueing systems in which customers arrive according to a Poisson process and are served according to a general distributed service time $(M / G / \cdot)$ plays a pivotal role in queueing theory. Under a set of assumptions, several analysis techniques have been developed for their transient and steady-state solution. For instance, in [8] a detailed analysis

Permission to make digital or hard copies of all or part of this work for personal or classroom use is granted without fee provided that copies are not made or distributed for profit or commercial advantage and that copies bear this notice and the full citation on the first page. To copy otherwise, to republish, to post on servers or to redistribute to lists, requires prior specific permission and/or a fee.

VALUETOOLS 2011, May 16-20, Paris, France

Copyright (c) 2011 ICST 978-1-936968-09-1

DOI 10.4108/icst.valuetools.2011.245722 of $M / G / 1$ queues is carried on. In this context, matrix analytic methods $[12,14]$ exploit the regular structure of the infinitesimal generator of the Continuous Time Markov Chain (CTMC) underlying a class of $M / G / 1$ queues (even with non-homogeneous arrival processes) in order to efficiently derive their steady-state probabilities. In this paper, we focus our attention on queueing systems of type $M / \mathrm{HYPO}_{K} / 1$ and $M / \mathrm{E}_{K} / 1$, i.e., whose arrivals occur according to a Poisson process, and service times are distributed according to a Hypo-exponential or Erlang distribution with $K$ exponential stages of service. Furthermore, we assume uncorrelated service times and independence between service times and the arrival process. This class of queues has a great importance in queueing theory and is characterised by the property of having a coefficient of variation of the service time that is less than 1. In literature, the analysis basically relies on the application of the Matrix Geometric technique or the application of the matrix explicit formula given in [13, 9]. Both these methods state the solution for the steady-state probabilities in terms of the matrix-blocks which the infinitesimal generator consists of.

Matrix Geometric has proved to be a powerful method for analysing many classes of queueing systems with very general arrival processes and service time distributions. The computation of the steady-state probabilities strongly depends on the knowledge of the rate matrix $\mathbf{R}$ whose analytical expression is known only for few cases. In general, matrix $\mathbf{R}$ must be computed by applying the iterative scheme presented in [12] or, for Quasi Birth and Death (QBD) processes, the one presented in [10]. The termination criterion for these iterative schemes depends on the definition of the desired numerical precision. We briefly review this method (especially to introduce the notation) in Section 2.1.

Alternatively, in $[13,9]$ the authors prove an efficient approach for the computation of matrix $\mathbf{R}$ that applies to queues which exhibit a specific block structure. According to this result, it suffices to compute the numerical inversion of an efficiently computable matrix (see Section 2.3 for a more detailed review).

The main contribution of this paper consists in giving an analytical expression for the rate matrix $\mathbf{R}$ required by the matrix analytic method applied to $M / \mathrm{HYPO}_{K} / 1$ (and, hence also $\left.M / \mathrm{E}_{K} / 1\right)$ queues. The knowledge of such an analytical expression is not only interesting from a theoretical point view, but allows also for a more efficient computation of ma- 
trix $\mathbf{R}$ (and, hence, of the steady-state probabilities) without the need of setting a tolerance criterion or without incurring the possible numerical problems due to a matrix-inversion operation. Furthermore, we exploit these results to provide a product-form approximation of $M / \mathrm{HYPO}_{K} / 1$ queues in a similar fashion of $[4,1]$. We consider a $M / \mathrm{HYPO}_{K} / 1$ queue feeding an infinite capacity queue in a tandem configuration. If the $M / \mathrm{HYPO}_{K} / 1$ queue has only one stage of service, then Burke's theorem can be applied to derive a product-form solution, because the behaviour of the first queue is independent of the state of the second one. However, if the first queue has more than one stage of service, to the best of our knowledge, no product-form solutions are known in literature. In fact, it can be easily shown that $M / \mathrm{HYPO}_{K} / 1$ queues, $K>1$, are neither quasi-reversible nor satisfy the Reversed Compound Agent Theorem (RCAT) conditions [3, 11] and these may be considered the main results that can be used to derive the product-form solution of a stochastic model. It is worthwhile noting that product-form solutions (even though approximated models) are important because they allow one to efficiently derive a set of average performance indices such as the mean number of customers in a queue, the mean response time, etc., but also detailed ones such as the response time distribution. In particular, in [4] the authors prove a very efficient approach to the computation of the response time distribution that may be applied also to the approximations given here. In this paper, we show that the knowledge of the expression of matrix $\mathbf{R}$ allows for the definition of more general approximations than those proposed in these papers and the non-linear optimization approach adopted in [1] is not needed anymore. When possible, the product-form approximations obtained in this paper are compared with that obtained in [4] and with the non-approximated queue.

The paper is structured as follows. Section 2 gives the basic notions and notations to keep the paper self-contained: Section 2.1 introduces the Matrix Geometric approach for the solution of QBD processes, Section 2.2 shows its application to $M / \mathrm{HYPO}_{K} / 1$ queues, and Section 2.3 briefly reviews the explicit matrix-formula for the computation of matrix $\mathbf{R}$ presented in $[13,9]$. Section 3 gives the symbolic expression for matrix $\mathbf{R}$ in case of $M / \mathrm{HYPO}_{K} / 1$ queues and proves the correctness of this result. An application to product-form analysis is presented in Section 4. Finally, Section 5 is devoted to some final remarks.

\section{THEORETICAL BACKGROUND}

In this part we recall the fundamental notions required to keep this paper self-contained. We briefly present Neuts' Matrix Geometric method and then we illustrate its standard application to $M / \mathrm{HYPO}_{K} / 1$ queues. In Section 2.3 we illustrate the (probably overlooked) result stated in [13] as clearly presented in [9].

\subsection{Neuts' Matrix Geometric method}

Matrix Geometric $[12,14]$ is an analysis technique that allows the efficient computation of the steady-state solution of structured Markov chains. In this paper we refer to the case of CTMCs whose infinitesimal generators $\mathbf{Q}$ are infinite block tridiagonal matrices with the following structure:

$$
\mathbf{Q}=\left(\begin{array}{ccccccc}
\mathbf{B}_{00} & \mathbf{B}_{01} & \mathbf{0} & \mathbf{0} & \mathbf{0} & \mathbf{0} & \ldots \\
\mathbf{B}_{10} & \mathbf{A}_{1} & \mathbf{A}_{2} & \mathbf{0} & \mathbf{0} & \mathbf{0} & \ldots \\
\mathbf{0} & \mathbf{A}_{0} & \mathbf{A}_{1} & \mathbf{A}_{2} & \mathbf{0} & \mathbf{0} & \ldots \\
\mathbf{0} & \mathbf{0} & \mathbf{A}_{0} & \mathbf{A}_{1} & \mathbf{A}_{2} & \mathbf{0} & \ldots \\
& & & \ddots & \ddots & \ddots & \\
\vdots & \vdots & \vdots & \vdots & \vdots & \vdots & \vdots
\end{array}\right)
$$

where:

- $\mathbf{A}_{0}, \mathbf{A}_{1}, \mathbf{A}_{2}$ are square matrices with the same dimension $\alpha$,

- $\mathbf{B}_{00}$ is a square matrix with dimension $\beta$,

- $\mathbf{B}_{10}$ is a $\alpha \times \beta$ matrix,

- $\mathbf{B}_{01}$ is a $\beta \times \alpha$ matrix.

Let $\boldsymbol{\pi}$ be the stationary distribution (assuming it exists) of the CTMC, i.e., $\boldsymbol{\pi} \mathbf{Q}=\mathbf{0}$ with the normalising condition $\boldsymbol{\pi} \mathbf{1}^{\top}=1$, where $\mathbf{1}=(1,1,1, \ldots)$. According to the structure of $\mathbf{Q}$, we consider $\boldsymbol{\pi}=\left(\boldsymbol{\pi}_{0}, \boldsymbol{\pi}_{1}, \boldsymbol{\pi}_{2}, \ldots\right)$ where $\boldsymbol{\pi}_{0}=$ $\left(\pi_{01}, \ldots, \pi_{0 \beta}\right)$ has dimension $\beta$, while $\boldsymbol{\pi}_{n}=\left(\pi_{n 1}, \ldots, \pi_{n \alpha}\right)$, $n>0$, has dimension $\alpha$. Vector $\pi$ may be iteratively computed with the following scheme:

$$
\boldsymbol{\pi}_{n+1}=\boldsymbol{\pi}_{n} \mathbf{R} \quad n>1,
$$

and $\boldsymbol{\pi}_{0}$ and $\boldsymbol{\pi}_{1}$ are defined as the solutions of:

$$
\left(\boldsymbol{\pi}_{0}, \boldsymbol{\pi}_{1}\right)\left(\begin{array}{cc}
\mathbf{B}_{00} & \mathbf{B}_{01} \\
\mathbf{B}_{10} & \mathbf{A}_{1}+\mathbf{R} \mathbf{A}_{0}
\end{array}\right)=(\mathbf{0}, \mathbf{0}),
$$

under the normalizing constraint:

$$
\boldsymbol{\pi}_{0} \mathbf{1}^{\top}+\boldsymbol{\pi}_{1}(\mathbf{I}-\mathbf{R})^{-1} \mathbf{1}^{\top}=1,
$$

where $\mathbf{I}$ is the identity matrix. In these expressions, matrix $\mathbf{R}$ plays a pivotal role and it must be the solution of the following equation:

$$
\mathbf{A}_{2}+\mathbf{R} \mathbf{A}_{1}+\mathbf{R}^{2} \mathbf{A}_{0}=\mathbf{0},
$$

with spectral radius strictly less than 1 . The numerical computation of $\mathbf{R}$ is an interesting problem which has been addressed by Neuts is his seminal work with the definition of an iterative scheme which has been proved to converge to the correct solution. This is straightforwardly derived from Equation (5). Let $\mathbf{R}_{(s)}$ be the matrix at the $s$-th iteration step, with $\mathbf{R}_{(0)}=\mathbf{0}$, then:

$$
\mathbf{R}_{(s+1)}=-\mathbf{A}_{2} \mathbf{A}_{1}^{-1}-\mathbf{R}_{(s)}^{2} \mathbf{A}_{0} \mathbf{A}_{1}^{-1}, \quad s \geq 0 .
$$

Neuts' scheme terminates when two successive iterations are close enough with respect to a given tolerance criterion. A faster iterative approach for the computation of $\mathbf{R}$ in case of QBD processes is described in [10]. However, finding explicit (symbolic) expressions for matrix $\mathbf{R}$ is often an important topic of research. Indeed, in general, the explicit computation of $\mathbf{R}$ is more efficient than the iterative schemes and does not suffer from numerical stability problems. Furthermore, the expression for $\mathbf{R}$ provides a deeper view on the relations among the steady-state probabilities of the considered model. 


\subsection{Matrix Geometric applied to $\mathrm{M} / \mathrm{Hypo} / 1$ queues}

In this section we apply the Matrix Geometric method to compute the steady-state solution of $M / \mathrm{HYPO}_{K} / 1$ queues. Although an explicit matrix formula which allows the efficient solution of this class of Markovian queues is available [13, 9], Matrix Geometric approach seems to be the favourite choice of many authors (see $[6,15]$ as an example). This is probably due to the fact that this approach is very versatile and can be easily applied to the case of phasetype distributed inter-arrival times. In $M / \mathrm{HYPO}_{K} / 1$ queue, stochastically identical customers arrive from the outside according to a homogeneous Poisson process with rate $\lambda$. At the arrival epoch the service starts immediately if the station is empty, or the customer waits in the queue according to First Come First Served policy, otherwise. We assume a hypo-exponential service time distribution with $K$ exponential stages, independent of the arrival process and uncorrelated (see [8] for a review of the properties of this random variable applied to queueing systems). Each stage of service has rate $\mu_{k}$, with $1 \leq k \leq K$ (see Figure 1 ). The stability condition of such a queue is well-known:

$$
\frac{1}{\lambda}>\sum_{k=1}^{K} \frac{1}{\mu_{k}} .
$$

Hereafter, we consider stable systems. If $\mu_{1}=\mu_{2}=\ldots=$

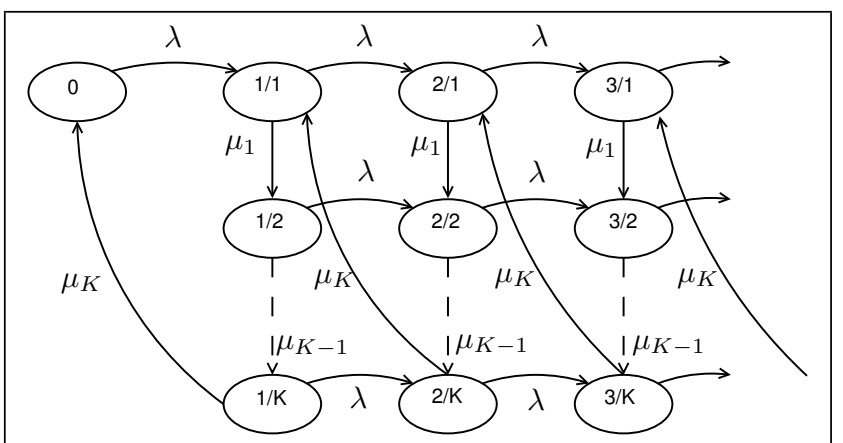

Figure 1: CTMC of a $M / \mathrm{HYPO}_{K} / 1$ queue with $K$ exponential stages.

$\mu_{K}=\mu$ then the service time distribution is modelled by an Erlang random variable with $K$ stages and rate $\mu / K$, i.e., the system becomes a $M / \mathrm{E}_{K} / 1$. It is well-know that this class of queueing systems has an underlying QBD process [14] that may be conveniently studied by the Matrix Geometric technique described in Section 2.1. Specifically, the structures of the matrices are the following (consult Figure 1):

$$
\begin{array}{ll}
\mathbf{B}_{00}=-\lambda & \\
\left(\mathbf{B}_{01}\right)_{1 j}=[j=1] \lambda, & 1 \leq j \leq K \\
\left(\mathbf{B}_{10}\right)_{i 1}=\left([i=K] \mu_{K}\right), & 1 \leq i \leq K \\
\left(\mathbf{A}_{1}\right)_{i j}=-[i=j]\left(\lambda+\mu_{i}\right)+[j=i+1] \mu_{i}, & 1 \leq i, j \leq K \\
\left(\mathbf{A}_{0}\right)_{i j}=[i=K][j=1] \mu_{K}, & 1 \leq i, j \leq K \\
\left(\mathbf{A}_{2}\right)_{i j}=[i=j] \lambda, & 1 \leq i, j \leq K
\end{array}
$$

where $(\mathbf{M})_{i j}$ denotes the element in row $i$ and column $j$ of matrix $\mathbf{M}$ and the square brackets denote the Iverson operator [5] applied to proposition $P$ :

$$
[P]= \begin{cases}1 & \text { if } P \\ 0 & \text { else. }\end{cases}
$$

(Iverson brackets)

For the $M / \mathrm{E}_{K} / 1$ queue, closed expression of the inverse of $\mathbf{A}_{1}$ is available, hence the iterative method for the computation of Neuts' matrix $\mathbf{R}$ may be simplified. The cases of queues with initial perturbations (e.g., with state-dependent arrival rates that yield a non-homogeneous Poisson process when the queue is in its initial states) differ only for the definition of matrices $\mathbf{B}_{01}, \mathbf{B}_{10}, \mathbf{B}_{00}$.

\subsection{Direct computation of matrix $R$}

Queueing theorists have traditionally directed many efforts in the analysis of $M / \mathrm{HYPO}_{K} / 1$ queues. A great improvement in the analysis methods of this class of queues is given in [13], where an explicit matrix-formula for the computation of matrix $\mathbf{R}$ is given. In this section we briefly review this result as stated in [9, Th. 8.5.1]. Let us consider a recurrent QBD process with the block-regular structure (1) and suppose that block $\mathbf{A}_{0}$ has rank 1. We can express $\mathbf{A}_{0}$ as the product of (row) vectors $\mathbf{c}$ and $\mathbf{r}: \mathbf{A}_{0}=\mathbf{c}^{\top} \mathbf{r}$. For $M / \mathrm{HYPO}_{K} / 1$ queues we have $\mathbf{c}=\left(0, \ldots, 0, \mu_{K}\right)$ and $\mathbf{r}=(1,0, \ldots, 0)$ (both vectors have size $K)$. In this case, matrix $\mathbf{R}$ is given by:

$$
\mathbf{R}=-\mathbf{A}_{2}\left(\mathbf{A}_{1}+\mathbf{A}_{2} \mathbf{1}^{\top} \mathbf{r}\right)^{-1} .
$$

REMARK 1. Note that in [9, Th. 8.5.1] the authors consider discrete-time $Q B D$ processes, whereas we consider continuous-time ones. Moreover, the theorem provides a characterization of the $G$ matrix from which equation (8) can be easily derived.

\section{SYMBOLIC SOLUTION OF M/HYPO/1 QUEUES}

In this section we define matrix $\mathbf{R}$ and prove that it is exactly the matrix obtained by the iterative algorithm based on recursive Equation (6) and that given by matrix-equation (8).

Definition 1 gives the symbolic expression for matrix $\mathbf{R}$. Table 1 provides some example of matrices obtained by the application of such a definition.

Definition 1. Let $\mathbf{R}=\left(r_{i j}\right), 1 \leq i, j \leq K$, be the matrix defined as follows:

$$
\begin{aligned}
r_{i j}=\frac{\lambda}{\mu_{j}} \prod_{k=j+1}^{i-1}( & \left.1+\frac{\lambda}{\mu_{k}}\right) \\
& \cdot\left\{\prod_{k=\max \{i, j+1\}}^{K}\left(1+\frac{\lambda}{\mu_{k}}\right)-[i>j]\right\} .
\end{aligned}
$$

Table 1 shows the structure of matrix $\mathbf{R}$ for $K=2,3,4$. In what follows, we prove that matrix $\mathbf{R}$ yields a set of important properties. Specifically, Lemma 1 introduces the expression for the inverse of matrix $\mathbf{R}$, and from this its 
- $K=2$

$$
\mathbf{R}=\left(\begin{array}{ll}
\frac{\lambda\left(\lambda+\mu_{2}\right)}{\mu_{1} \mu_{2}} & \frac{\lambda}{\mu_{2}} \\
\frac{\lambda^{2}}{\mu_{1} \mu_{2}} & \frac{\lambda}{\mu_{2}}
\end{array}\right)
$$

- $K=3$

$$
\mathbf{R}=\left(\begin{array}{ccc}
\frac{\lambda\left(\lambda+\mu_{2}\right)\left(\lambda+\mu_{3}\right)}{\mu_{1} \mu_{2} \mu_{3}} & \frac{\lambda\left(\lambda+\mu_{3}\right)}{\mu_{2} \mu_{3}} & \frac{\lambda}{\mu_{3}} \\
\frac{\lambda^{2}\left(\lambda+\mu_{2}+\mu_{3}\right)}{\mu_{1} \mu_{2} \mu_{3}} & \frac{\lambda\left(\lambda+\mu_{3}\right)}{\mu_{2} \mu_{3}} & \frac{\lambda}{\mu_{3}} \\
\frac{\lambda^{2}\left(\lambda+\mu_{2}\right)}{\mu_{1} \mu_{2} \mu_{3}} & \frac{\lambda^{2}}{\mu_{2} \mu_{3}} & \frac{\lambda}{\mu_{3}}
\end{array}\right)
$$

- $K=4$

$$
\mathbf{R}=\left(\begin{array}{cccc}
\frac{\lambda\left(\lambda+\mu_{2}\right)\left(\lambda+\mu_{3}\right)\left(\lambda+\mu_{4}\right)}{\mu_{1} \mu_{2} \mu_{3} \mu_{4}} & \frac{\lambda\left(\lambda+\mu_{3}\right)\left(\lambda+\mu_{4}\right)}{\mu_{2} \mu_{3} \mu_{4}} & \frac{\lambda\left(\lambda+\mu_{4}\right)}{\mu_{3} \mu_{4}} & \frac{\lambda}{\mu_{4}} \\
\frac{\lambda\left(\lambda^{3}+\lambda^{2}\left(\mu_{2}+\mu_{3}+\mu_{4}\right)+\lambda\left(\mu_{2} \mu_{3}+\mu_{3} \mu_{4}+\mu_{2} \mu_{4}\right)\right)}{\mu_{1} \mu_{2} \mu_{3} \mu_{4}} & \frac{\lambda\left(\lambda+\mu_{3}\right)\left(\lambda+\mu_{4}\right)}{\mu_{2} \mu_{3} \mu_{4}} & \frac{\lambda\left(\lambda+\mu_{4}\right)}{\mu_{3} \mu_{4}} & \frac{\lambda}{\mu_{4}} \\
\frac{\lambda\left(\lambda+\mu_{2}\right)\left(\lambda+\mu_{3}+\mu_{4}\right)}{\mu_{1} \mu_{2} \mu_{3} \mu_{4}} & \frac{\lambda^{2}\left(\lambda+\mu_{3}+\mu_{4}\right)}{\mu_{2} \mu_{3} \mu_{4}} & \frac{\lambda\left(\lambda+\mu_{4}\right)}{\mu_{3} \mu_{4}} & \frac{\lambda}{\mu_{4}} \\
\frac{\lambda^{2}\left(\lambda+\mu_{2}\right)\left(\lambda+\mu_{3}\right)}{\mu_{1} \mu_{2} \mu_{3} \mu_{4}} & \frac{\lambda^{2}\left(\lambda+\mu_{3}\right)}{\mu_{2} \mu_{3} \mu_{4}} & \frac{\lambda^{2}}{\mu_{3} \mu_{4}} & \frac{\lambda}{\mu_{4}}
\end{array}\right)
$$

determinant may be easily derived as done in Corollary 1 . The former Lemma, although interesting in itself, plays a pivotal role in the proof of the main result of the paper, Theorem 1 (and also Theorem 2).

LEMma 1 (INVERSE OF $\mathbf{R}$ ). Matrix $\mathbf{R}^{-1}=\left(q_{i j}\right)$, where $1 \leq i, j \leq K$, which is defined as:

$$
q_{i j}=-[j=1]+[i=j]\left(1+\frac{\mu_{i}}{\lambda}\right)-[j=i+1] \frac{\mu_{i}}{\lambda}
$$

is the inverse of $\mathbf{R}$.

ProOF. First, we prove that:

$$
r_{i j}-r_{(i+1) j}=\frac{\lambda}{\mu_{i}}\left([i=j]+r_{1 j}-r_{i j}\right) .
$$

The expression of $r_{1 j}-r_{i j}$ in the right-hand-side is:

$$
\begin{array}{r}
r_{1 j}-r_{i j}=\frac{\lambda}{\mu_{j}} \prod_{k=j+1}^{K}\left(1+\frac{\lambda}{\mu_{k}}\right)-\frac{\lambda}{\mu_{j}} \prod_{k=j+1}^{i-1}\left(1+\frac{\lambda}{\mu_{k}}\right) \\
\cdot\left\{\prod_{k=\max \{i, j+1\}}^{K}\left(1+\frac{\lambda}{\mu_{k}}\right)-[i>j]\right\} \\
=\frac{\lambda}{\mu_{j}} \prod_{k=j+1}^{i-1}\left(1+\frac{\lambda}{\mu_{k}}\right)[i>j] .
\end{array}
$$

The left-hand-side of Equation (12) can be written as follows:

$$
\begin{gathered}
r_{i j}-r_{(i+1) j}=\frac{\lambda}{\mu_{j}} \prod_{k=j+1}^{i-1}\left(1+\frac{\lambda}{\mu_{k}}\right)\left\{\prod_{k=i}^{K}\left(1+\frac{\lambda}{\mu_{k}}\right)[i>j]\right. \\
+\prod_{k=j+1}^{K}\left(1+\frac{\lambda}{\mu_{k}}\right)[i \leq j]-[i>j]-\left(\left(1+\frac{\lambda}{\mu_{i}}\right)[j<i]+[j \geq i]\right) \\
\left.\cdot\left(\prod_{k=\max \{j+1, i+1\}}^{K}\left(1+\frac{\lambda}{\mu_{k}}\right)-[i \geq j]\right)\right\} .
\end{gathered}
$$

By the properties of Iverson brackets, this reduces to:

$$
\begin{array}{r}
\frac{\lambda}{\mu_{j}} \prod_{k=j+1}^{i-1}\left(1+\frac{\lambda}{\mu_{k}}\right)\left\{[ i > j ] \left(\prod_{k=i}^{K}\left(1+\frac{\lambda}{\mu_{k}}\right)-1\right.\right. \\
\left.\left.-\left(1+\frac{\lambda}{\mu_{i}}\right) \prod_{k=i+1}^{K}\left(1+\frac{\lambda}{\mu_{k}}\right)+\left(1+\frac{\lambda}{\mu_{i}}\right)\right)+[i=j]\right\} \\
=\frac{\lambda}{\mu_{j}} \prod_{k=j+1}^{i-1}\left(1+\frac{\lambda}{\mu_{k}}\right)\left(\frac{\lambda}{\mu_{i}}[i>j]+[i=j]\right) .
\end{array}
$$

After some algebra, we obtain:

$$
\begin{aligned}
\frac{\lambda}{\mu_{i}}\left(\frac{\lambda}{\mu_{j}} \prod_{k=j+1}^{i-1}\left(1+\frac{\lambda}{\mu_{k}}\right)[i>j]\right. & +[i=j]) \\
& =\frac{\lambda}{\mu_{i}}\left([i=j]+r_{1 j}-r_{i j}\right),
\end{aligned}
$$

as required to prove Equation (12). In order to conclude the proof of the Lemma, we show that $\mathbf{R}^{-1} \mathbf{R}=I$, where $I$ is the identity matrix. Let $\left(\mathbf{R}^{-1} \mathbf{R}\right)_{i j}$, with $1 \leq i, j \leq K$, denote the $i, j$ element of $\mathbf{R}^{-1} \mathbf{R}$, then we have:

$$
\begin{aligned}
\left(\mathbf{R}^{-1} \mathbf{R}\right)_{i j}= & \sum_{\ell=1}^{K} q_{i \ell} r_{\ell j} \\
= & \sum_{\ell=1}^{K}\left\{-[\ell=1]+[i=\ell]\left(1+\frac{\mu_{i}}{\lambda}\right)\right. \\
& \left.-[\ell=i+1] \frac{\mu_{i}}{\lambda}\right\} r_{\ell j} \\
= & -r_{1 j}+\left(1+\frac{\mu_{i}}{\lambda}\right) r_{i j}-\frac{\mu_{i}}{\lambda} r_{(i+1) j} \\
= & r_{i j}-r_{1 j}+\frac{\mu_{i}}{\lambda}\left(r_{i j}-r_{(i+1) j}\right)=[i=j] .
\end{aligned}
$$

From $\mathbf{R}^{-1}$ we may easily derive the determinant of $\mathbf{R}$ as proved by the following corollary: 
Corollary 1 (Determinant of $\mathbf{R}$ ). The determinant of $\mathbf{R}$ is:

$$
\operatorname{det}(\mathbf{R})=\prod_{k=1}^{K} \frac{\lambda}{\mu_{k}}
$$

Proof. We proceed by finding the determinant of $\mathbf{R}^{-1}$, which has a simpler form than $\mathbf{R}$. Its inverse will then provide us with the determinant of $\mathbf{R}$.

Let $\mathbf{B}=\left(b_{i j}\right)$ be the matrix defined as $b_{i j}=[i \leq j]$. This is an upper triangular matrix with determinant 1 . Then,

$$
\begin{aligned}
\left(\mathbf{R}^{-1} \mathbf{B}\right)_{i j} & =\sum_{\ell=1}^{K} q_{i \ell}[\ell \leq j] \\
& =-[j \geq 1]+\left(1+\frac{\mu_{i}}{\lambda}\right)[i \leq j]-\frac{\mu_{i}}{\lambda}[i+1 \leq j] \\
& =-1+\left(1+\frac{\mu_{i}}{\lambda}\right)[i \leq j]-\frac{\mu_{i}}{\lambda}([i \leq j]-[i=j]) \\
& =-1+[i \leq j]+\frac{\mu_{i}}{\lambda}[i=j]=t_{i j} .
\end{aligned}
$$

Note that matrix $\mathbf{T}=\left(t_{i j}\right)$ is a lower triangular matrix with $i$ th diagonal element given by $\mu_{i} / \lambda$. The determinant of $T$ is thus simply given by

$$
\operatorname{det}(\mathbf{T})=\operatorname{det}\left(\mathbf{R}^{-1} \mathbf{B}\right)=\operatorname{det}\left(\mathbf{R}^{-1}\right)=\prod_{i=1}^{K} \frac{\mu_{i}}{\lambda} .
$$

The result follows as

$$
\operatorname{det}(\mathbf{R})=\operatorname{det}\left(\mathbf{R}^{-1}\right)^{-1}=\operatorname{det}(\mathbf{T})^{-1}=\prod_{i=1}^{K} \frac{\lambda}{\mu_{i}} .
$$

The following theorem is fundamental because it shows that matrix $\mathbf{R}$ given by Definition 1 coincides with that obtained by the iterative computation (6) and by Equation (8). Its proof relies on showing that matrix $\mathbf{R}$ given by Lemma 1 is identical to the expression in Equation (8).

Theorem 1. The matrix $\mathbf{R}$ from Definition 1 is the rate matrix for $M / \mathrm{HYPO}_{K} / 1$ queues.

Proof. The result follows from [9, Th. 8.5.1] by showing that Equation (8) holds when $\mathbf{R}$ is defined as in Definition 1. For simplicity, however, we will equivalently show that $\mathbf{R}^{-1}$, i.e., the inverse of $\mathbf{R}$ from Lemma 1, satisfies

$$
\mathbf{R}^{-1}=-\left(\mathbf{A}_{1}+\mathbf{A}_{2} \mathbf{1}^{\top} \mathbf{r}\right) \mathbf{A}_{2}^{-1} .
$$

This can be straightforwardly seen by writing it in terms of the Iverson bracket notation. Indeed,

$$
\begin{aligned}
\left(\mathbf{R}^{-1}\right)_{i j}=\frac{1}{\lambda}([i=j] & \left(\lambda+\mu_{i}\right) \\
- & {\left.[j=i+1] \mu_{i}-\lambda[j=1]\right)=q_{i j} . }
\end{aligned}
$$

Additionally, in appendix we provide an alternative and selfcontained proof showing that the expression of $\mathbf{R}$ given by Definition 1 satisfies Equation (5) and is that with spectral radius strictly lower than 1 .

\section{APPLICATION TO PRODUCT-FORM}

In this section, we apply the results on $M / \mathrm{HYPO}_{K} / 1$ queues to carry out an approximate product-form analysis. It is well-known that $M / \mathrm{HYPO}_{K} / 1$ queues are not quasi-reversible [7] when $K>1$ and, hence, an exact product-form solution is not known in literature. However, as observed in [4], we may consider a modified version of $M / \mathrm{HYPO}_{K} / 1$ queues that yields the quasi-reversibility property. In [4] the authors consider a tandem of a $M / \mathrm{E}_{2} / 1$ queue with a $\cdot / M / 1$ queue (see Figure 2) and show that providing a product-form approximation of such a system allows for the derivation of both average and detailed performance indices (specifically, the paper presents an efficient novel technique for the computation of the response time distribution). In this example we show

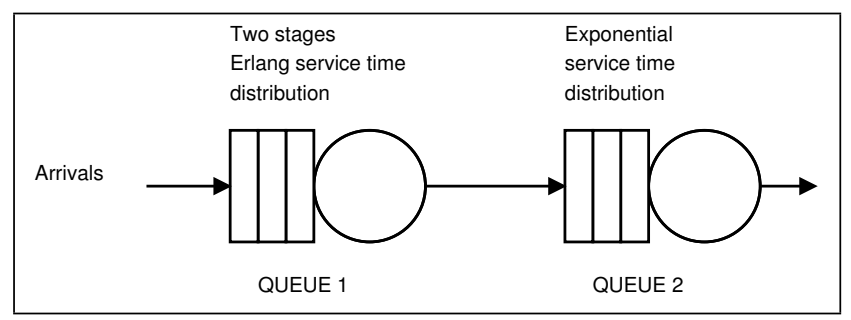

Figure 2: Tandem of a queue with Erlang service time distribution (Queue 1) and a queue with exponential service time distribution (Queue 2).

how it is possible to extend the product-form approximation approach of [4] to queues with Hypo-exponential service time distributions with arbitrary number of stages. Consider the underlying stochastic process of a $M / \mathrm{HYPO}_{K} / 1$ queue as depicted by Figure 3 in which the dotted arcs represent the perturbations introduced to obtain a product-form model from the original one. The goal of the analysis consists in finding the correct rates of $\lambda_{1}, \ldots, \lambda_{K}$ (where $K$ is the number of stages of the hypo-exponential queue), $\nu, \mu_{11}, \ldots$, $\mu_{1 K}, \sigma$ and $\sigma_{0}$ in order to have that the reversed rates of the transitions corresponding to customer departures (those with rates $\left.\mu_{K}\right)$ are independent of the departure/arriving state (i.e., they are constant). Let $\bar{d}$ be this reversed rate. The model depicted by Figure 3 satisfies the structural and rate conditions of the Reversed Compound Agent Theorem (RCAT) [3] and, hence, its composition with other models yielding the same properties has product-form stationary distribution.

REMARK 2. Note that, according to the methodology developed in [4] the arrival events at Queue 2 are synchronised with the transitions with rate $\mu_{k}$ and with the self-loops with rate $\sigma$. In this paper we have also added a self-loop in state 0 with rate $\sigma_{0}$ and this implies that this state has two incoming transitions which synchronise with the arrivals at the second queue. It has been proved in [11] that in this cases the sum of the reversed rates coming into state 0 must be equal to $\bar{d}$. 


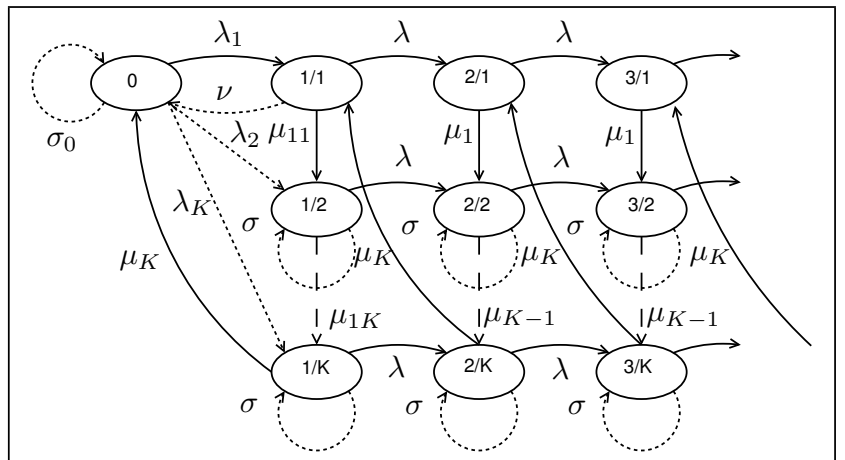

Figure 3: Perturbations on $M / \mathrm{HYPO}_{K} / 1$ queues in order to yield product-form conditions stated by RCAT.

Let us consider the general problem of finding the conditions on the free rates $\lambda_{1}, \ldots, \lambda_{K}, \nu, \mu_{11}, \ldots, \mu_{1 K}$ in order to have a state-independent reversed rate for the departure transitions. By $[7,3]$, the reversed rate of the departure transition from state $(n+1, K)$ to state $(n, 1)$ is:

$$
\bar{d}(n)=\frac{\pi_{(n+1) K}}{\pi_{n 1}} \mu_{K}, \quad n \geq 0,
$$

where $\pi_{01} \equiv \pi_{0}$. We require that:

$$
\bar{d}(n)=\bar{d} \text { for all } n>0 \wedge \bar{d}(0)+\sigma_{0}=\bar{d} .
$$

By Theorem 2 we have $\boldsymbol{\pi}_{n+1}=\boldsymbol{\pi}_{n} \mathbf{R}$ and, since $\mathbf{R}$ is nonsingular we straightforwardly have $\boldsymbol{\pi}_{n}=\boldsymbol{\pi}_{n+1} \mathbf{R}^{-1}$. Hence:

$$
\begin{aligned}
& \bar{d}=\mu_{K} \frac{\pi_{(n+1) K}}{\pi_{(n+1) 1} \frac{\mu_{1}}{\lambda}-\sum_{j=2}^{K} \pi_{(n+1) j}} \\
&=\mu_{K} \frac{\alpha_{K}(n+1)}{\frac{\mu_{1}}{\lambda}-\sum_{j=2}^{K} \alpha_{j}(n+1)}, \quad n>0,
\end{aligned}
$$

where $\alpha_{j}(n)=\pi_{n j} / \pi_{n 1}$. A sufficient condition for Equation (13) to hold is that for all $n>0$ :

$$
\alpha_{j}(n)=\alpha_{j}
$$

Therefore, we can write:

$$
\alpha_{j}=\frac{\pi_{n j}}{\pi_{n 1}}=\frac{-\pi_{(n+1)(j-1)} \frac{\mu_{j-1}}{\lambda}+\pi_{(n+1) j}\left(1+\frac{\mu_{j}}{\lambda}\right)}{\pi_{(n+1) 1} \frac{\mu_{1}}{\lambda}-\sum_{i=2}^{K} \pi_{(n+1) i}}
$$

for $n>0$ and $j>1$. Dividing both numerator and denominator by $\pi_{(n+1), 1}$, after some algebra we obtain the following system of equations on $\alpha_{j}$ :

$$
\left\{\begin{array}{l}
\alpha_{1}=1 \\
\lambda \alpha_{j}^{2}+\alpha_{j}\left(\lambda \sum_{\substack{i=1 \\
i \neq j}}^{K} \alpha_{i}+\mu_{j}-\mu_{1}\right) \\
-\alpha_{j-1} \mu_{j-1}=0, \quad 2 \leq j \leq K .
\end{array}\right.
$$

This system of quadratic equations can be easily solved numerically as stated by Lemma 2 or, in case of few stages (and, hence, unknowns), even symbolically via the computation of the Gröbner basis [2].
Lemma 2. Let $t_{0}=\mu_{1}-\min _{j=1 \ldots K} \mu_{i}$ and consider the following single-variate function

$$
f(t)=-t+\lambda \sum_{i=1}^{K} \prod_{j=2}^{i} \frac{\mu_{j-1}}{t+\mu_{j}-\mu_{1}} .
$$

The following propositions hold:

1. $f(t)$ is continuous and monotonically decreasing in the interval $\left(t_{0},+\infty\right)$;

2. $f(t)$ has a unique zero $t^{*}$ in the same interval;

3. the unique feasible solution of System (16), i.e., such that $\alpha_{j}>0$ for all $j=1 \ldots K$, can be computed through the recursive formula:

$$
\alpha_{j}= \begin{cases}\alpha_{j-1} \frac{\mu_{j-1}}{t^{*}+\mu_{j}-\mu_{1}} & 2 \leq j \leq K \\ 1 & j=1\end{cases}
$$

Proof. We start by proving the first proposition. Function $f(t)$ is trivially continuous in $\left(t_{0},+\infty\right)$ since all discontinuities are located at values of $t \leq t_{0}$. Moreover, it is monotonically decreasing because

$$
f^{\prime}(t)=-\left[1+\lambda \sum_{i=1}^{K} \sum_{\ell=2}^{i} \frac{1}{t+\mu_{\ell}-\mu_{1}} \prod_{j=2}^{i} \frac{\mu_{j-1}}{t+\mu_{j}-\mu_{1}}\right]<0,
$$

for $t>t_{0}$.

The second proposition follows from the observation that $\lim _{t \rightarrow t_{0}^{+}} f(t)=+\infty$ and $\lim _{t \rightarrow+\infty} f(t)=-\infty$. Indeed, this fact combined with the first proposition implies the existence of a unique zero $t^{*}$ in the interval $\left(t_{0},+\infty\right)$.

Finally, we prove the third proposition. Let us write:

$$
t=\lambda \sum_{i=1}^{K} \alpha_{i}
$$

By considering this relation, we can derive from System (16) the following recursive formula for the $\alpha$ 's:

$$
\alpha_{j}= \begin{cases}\alpha_{j-1} \frac{\mu_{j-1}}{t+\mu_{j}-\mu_{1}} & 2 \leq j \leq K \\ 1 & j=1\end{cases}
$$

By unfolding the recursion we obtain

$$
\alpha_{j}=\prod_{i=2}^{j} \frac{\mu_{i-1}}{t+\mu_{i}-\mu_{1}},
$$

and by substituting this into (19) we end up with the following equation in $t$ :

$$
-t+\lambda \sum_{i=1}^{K} \prod_{j=2}^{i} \frac{\mu_{j-1}}{t+\mu_{j}-\mu_{1}}=0 .
$$

Note that $f(t)$ in (17) is the left-hand side of this equation and that any zero $\tau$ of it allows us to recover a solution of System (16) by applying (20) (with $t$ replaced by $\tau$ ). However, in order to have a feasible solution, i.e., which guarantees to have positive $\alpha$ 's, a necessary and sufficient condition for the zero is to lie in the interval $\left(t_{0},+\infty\right)$. The proof now follows from the first and second propositions. 
REMARK 3. Notice that the desirable properties of function $f(t)$ proved in Lemma 2 allow for an efficient numerical computation of the solution of System (16). For instance, one may employ section search algorithms over the interval $\left(t_{0},+\infty\right)$ with a convergence guarantee to the correct solution.

Note that, once the values of $\alpha_{1}, \ldots, \alpha_{K}$ are known, one may easily compute the reversed rate $\bar{d}$ by Equation (14):

$$
\bar{d}=\frac{\lambda \alpha_{K} \mu_{K}}{\mu_{1}-\lambda \sum_{j=2}^{K} \alpha_{j}} .
$$

We may also express the steady-state probabilities for $n \geq 2$ as stated by the following Lemma.

Lemma 3. The steady-state probabilities for the perturbed $M / \mathrm{HYPO}_{K} / 1$ queue satisfying Condition (15) -and, hence, also Condition (14)- for $n \geq 2$ are given by the following expression:

$$
\pi_{n k}=\alpha_{k}\left(\frac{\lambda}{\mu_{1}-\lambda \sum_{j=2}^{K} \alpha_{j}}\right)^{n-1} \pi_{11} .
$$

Proof. We proceed by induction on $n$ considering as the base case $n=1$. We have:

$$
\pi_{1 k}=\alpha_{k} \pi_{11} .
$$

Note that $\alpha_{k}(1)=\pi_{1 k} / \pi_{11}$ and by hypothesis $\alpha_{k}(1)=\alpha_{k}$. Let us consider $n>1$, we can write:

$$
\begin{aligned}
\pi_{n k}=\frac{\pi_{n k}}{\pi_{n 1}} \frac{\pi_{n 1}}{\pi_{n K}} \frac{\pi_{n K}}{\pi_{(n-1) 1}} \frac{\pi_{(n-1) 1}}{\pi_{(n-1) K}} \pi_{(n-1) K} \\
=\frac{\alpha_{k}}{\alpha_{K}} \frac{\bar{d}}{\alpha_{K} \mu_{K}} \pi_{(n-1) K}
\end{aligned}
$$

Now, by applying the inductive hypotheses, we obtain:

$$
\begin{aligned}
\pi_{n k}=\frac{\alpha_{k}}{\alpha_{K}} \frac{\bar{d}}{\alpha_{K} \mu_{K}} \alpha_{K} & \left(\frac{\lambda}{\mu_{1}-\lambda \sum_{j=2}^{K} \alpha_{j}}\right)^{n-2} \pi_{11} \\
& =\alpha_{k}\left(\frac{\lambda}{\mu_{1}-\lambda \sum_{j=2}^{K} \alpha_{j}}\right)^{n-1} \pi_{11},
\end{aligned}
$$

as required.

In order to find the correct parametrisation of the model, i.e., a set of values for $\lambda_{1}, \ldots, \lambda_{K}, \mu_{11}, \ldots, \mu_{1 K}$ and $\nu$ that satisfies Condition (13), we must solve the system of equations (3) under its normalising constraint combined with the equations $\pi_{1 k}=\alpha_{k} \pi_{11}$ for $k=2, \ldots, K$. Note that the number of parameters allows one to choose among different approximated models. In order to satisfy RCAT conditions we must have $\sigma=\bar{d}$ and $\sigma_{0}=\bar{d}-\mu_{K} \pi_{1 K} / \pi_{0}$ since the reversed rate of a self-loop is identical to its forward rate [3].

\subsection{A numerical example}

We consider a queue under homogeneous arrivals generated according to a Poisson process and independent service time distributed according to a hypo-exponential random variable with 5 stages (numerical values are given in Table 2).

\begin{tabular}{|l|l|}
\hline Parameter & Value \\
\hline$\lambda$ & 0.33333 \\
$\mu_{1}$ & 2.0000 \\
$\mu_{2}$ & 2.5000 \\
$\mu_{3}$ & 1.0000 \\
$\mu_{4}$ & 6.0000 \\
$\mu_{5}$ & 3.0000 \\
\hline
\end{tabular}

Table 2: Numerical instances of the queue parameters studies in Section 4.1.

System of polynomial equations (16) can be easily solved numerically, e.g., by applying the result in Lemma 2. Figure 4 shows the plot of function $f(t)$ for this numerical example. As one can see there is a zero after the last asymptote, which is indeed unique as stated by the lemma. From this

Plotting of function $f(t)$ for the numerical example with $K=5$

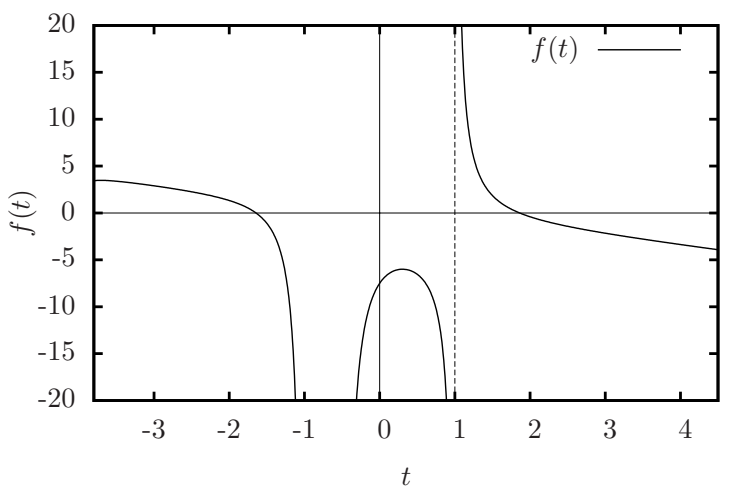

Figure 4: Plotting of function $f(t)$ as defined by Equation (17) for the numerical example of Section 4.1.

we obtain the unique feasible solution shown in Table 3.

\begin{tabular}{|l|l|}
\hline Parameter & Value \\
\hline$\alpha_{1}$ & 1.0000 \\
$\alpha_{2}$ & 0.84624 \\
$\alpha_{3}$ & 2.4503 \\
$\alpha_{4}$ & 0.41791 \\
$\alpha_{5}$ & 0.87569 \\
\hline
\end{tabular}

Table 3: Numerical solution of System (16) for the example of Section 4.1.

We can now compute the reversed rate $\bar{d}$ by Equation (14), which turns out to be 1.86339 . Now, we find the definition of the initial perturbation. We set $\lambda_{2}=\ldots=\lambda_{K}=\nu=0$. We also add as a constraint $\pi_{0}=1-\lambda\left(\mu_{1}^{-1}+\ldots+\mu_{5}^{-1}\right)$, i.e., in the modified model satisfying RCAT, the probability of observing the empty queue must not be changed. Therefore, by solving linear system (3) -combined with the normalizing condition- we obtain the set of parameters of Table 4, and the steady-state probabilities $\boldsymbol{\pi}_{1}$ of Table 5 .

Figure 5 shows a comparison between the steady-state prob- 


\begin{tabular}{|l|l|}
\hline Parameter & Value \\
\hline$\mu_{11}$ & 4.1571 \\
$\mu_{12}$ & 4.5791 \\
$\mu_{13}$ & 1.2481 \\
$\mu_{14}$ & 6.9847 \\
$\lambda_{1}$ & 0.54643 \\
$\sigma_{0}$ & 1.3170 \\
\hline
\end{tabular}

Table 4: Parameters of the product-form model.

\begin{tabular}{|l|l|}
\hline Component & Stationary probability \\
\hline$\pi_{11}$ & 0.041600 \\
$\pi_{12}$ & 0.035204 \\
$\pi_{13}$ & 0.10193 \\
$\pi_{14}$ & 0.017385 \\
$\pi_{15}$ & 0.036428 \\
\hline
\end{tabular}

Table 5: Steady-state probabilities $\pi_{1}$ of the modified model.

ability of observing $j$ customers $\left(\pi_{j}=\sum_{k=1}^{K} \pi_{j k}, j>0\right)$ in the $M / \mathrm{HYPO}_{K} / 1$ queue and its product-form approximation. As one can see the product-form model obtained by applying the described approximation technique is very tight to the corresponding non-approximated $M / \mathrm{HYPO}_{K} / 1$ queue in terms of the distribution of the total number of customers.

Aggregated steady-state probabilities of the queue

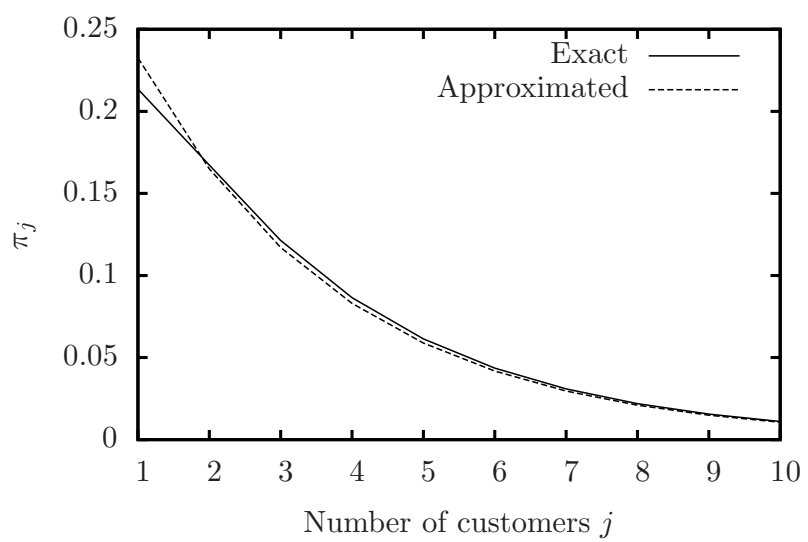

Figure 5: Comparison between the aggregated (for total number of customers) steady-state probabilities of the $M / \mathrm{HYPO}_{K} / 1$ queue and its product-form approximation.

\subsection{Application to the case of $K=2$ stages}

In our framework the case of $K=2$ stages becomes trivial because the system of polynomial equations (16) reduces to one quadratic equation:

$$
\lambda \alpha_{2}^{2}+\alpha_{2}\left(\lambda+\mu_{2}-\mu_{1}\right)-\mu_{1}=0
$$

whose unique positive solution is:

$$
\alpha_{2}=\frac{-\left(\lambda+\mu_{2}-\mu_{1}\right)+\sqrt{\Delta}}{2 \lambda},
$$

where $\Delta=\left(\lambda+\mu_{2}-\mu_{1}\right)^{2}+4 \lambda \mu_{1}$. We may also derive the expression for the reversed rate of the departure transitions by applying Equation (21), that is:

$$
\bar{d}=\frac{1}{2}\left(\lambda+\mu_{1}-\mu_{2}+\sqrt{\Delta}\right) .
$$

Notice that the result is coherent with that provided in [4] under the assumption $\mu_{1}=\mu_{2}=\mu^{1}$. Nevertheless, we can consider this result as a generalisation of the approximation presented in [4] because it holds even if $\mu_{2} \neq \mu_{1}$ (notice also that our approach does not require the solution of a third degree equation as in the previous one). Also in this case we aim to solve the boundary equation for the initial states in order to find a correct parametrisation of the initial perturbation. We impose the constraint that the steadystate probability of the empty queue in the original and in the approximated model must be the same, and this gives the following solution:

$$
\begin{aligned}
\lambda_{1}= & \frac{\lambda\left(\mu_{1}+\mu_{2}\right)\left(\lambda-\mu_{1}-\mu_{2}+\sqrt{\Delta}\right)}{2\left(\lambda\left(\mu_{1}+\mu_{2}\right)-\mu_{1} \mu_{2}\right)} \\
\mu_{11}= & \left(\lambda+\mu_{2}\right) \alpha_{2} \\
\sigma_{0}= & \frac{\mu_{1}\left(\lambda\left(2 \mu_{1}+\mu_{2}\right)+\mu_{2}\left(-\mu_{1}+\mu_{2}-\sqrt{\Delta}\right)\right)}{2\left(\lambda\left(\mu_{1}+\mu_{2}\right)-\mu_{1} \mu_{2}\right)} \\
\pi_{0}= & 1-\lambda\left(\frac{1}{\mu_{1}}+\frac{1}{\mu_{2}}\right) \\
\pi_{11}= & \pi_{0} \lambda\left(\mu_{1}+\mu_{2}\right) \frac{\left(\lambda-\mu_{1}-\mu_{2}+\sqrt{\Delta}\right)}{4 \mu_{1} \mu_{2}\left(\lambda\left(\mu_{1}+\mu_{2}\right)-\mu_{1} \mu_{2}\right)} \\
& \cdot\left(\lambda-\mu_{1}+\mu_{2}+\sqrt{\Delta}\right) \\
\pi_{12}= & \pi_{0} \lambda\left(\mu_{1}+\mu_{2}\right) \frac{\lambda-\mu_{1}-\mu_{2}+\sqrt{\Delta}}{2 \mu_{2}\left(\lambda\left(\mu_{1}+\mu_{2}\right)-\mu_{1} \mu_{2}\right)}
\end{aligned}
$$

Figure 6, 7, 8 show a comparison among the aggregate steadystate probabilities (i.e., the probability of observing $n$ customers in the queue despite to the stage of service) in the original model, in the approximation proposed in this paper, and in that proposed in [4] under different scenarios. The figures suggest that the perturbation method proposed here for $M / \mathrm{HYPO}_{K} / 1$ queues provides a tighter approximation than the previous one presented in [4].

\subsection{Final considerations}

It should be clear that the symbolical derivation of the productform approximation may result computational expensive as the number of stages of service (and, hence, the model parameters) grows. We have experimented that the case of $M / E_{3} / 1$ queue is still fully tractable symbolically. For this case System (16) becomes:

$$
\left\{\begin{array}{l}
\alpha_{1}=1 \\
\lambda \alpha_{2}^{2}+\lambda\left(\alpha_{1}+\alpha_{3}\right) \alpha_{2}-\mu=0 \\
\lambda \alpha_{3}^{2}+\lambda\left(\alpha_{1}+\alpha_{2}\right) \alpha_{3}-\alpha_{2} \mu=0
\end{array}\right.
$$

On the other hand, a fully numerical approach results very efficient. It is worthwhile noting that the method we presented allows the modeller to specify the values only for some ${ }^{1}$ In [4] the authors derive the expression for $\kappa=\pi_{12} / \pi_{0}=$ $\left(\lambda+\left(\lambda^{2}+4 \lambda \mu\right)^{1 / 2}\right) /(2 \mu)$. Note that $\kappa \mu$ is the reversed rate of the job-completion transition. 


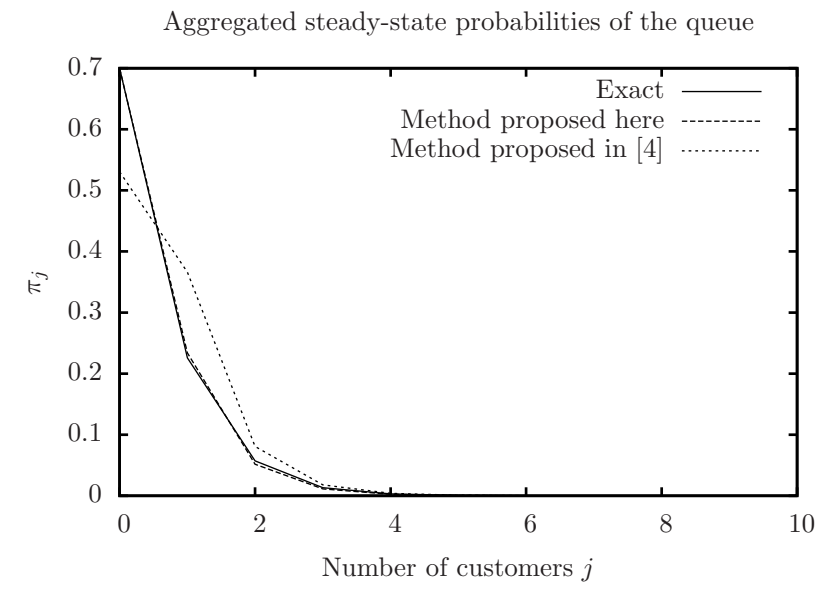

Figure 6: Comparison of aggregated steady-state probabilities of the $M / E_{2} / 1$ queue with its productform approximations. Parameters are $\lambda=0.3$ and $\mu=2$.

of the queue parameters and then derive the approximations. This may be useful in case of sensitivity analysis.

\section{CONCLUSION}

In this paper we have given the symbolic expression for matrix $\mathbf{R}$ which plays a pivotal role in matrix-analytic methods for the analysis of $M / \mathrm{HYPO}_{K} / 1$ queues. In literature, the analysis of this class of queueing systems relies on the application of the Matrix Geometric method with the opportune iterative algorithm $[12,10,15]$ or on a matrix inversion as proposed in $[13,9]$. Although both these methods are efficient and present good numerical properties, the explicit expression for rate matrix $\mathbf{R}$ may be useful in proofs in which the symbolic expression for steady-state probabilities are required. As an example we have shown how this theoretical result may be applied to the definition of product-form approximations of $M / \mathrm{HYPO}_{K} / 1$ queues. Specifically, we have generalised the results proposed in $[4,1]$ and shown how the exact solution for the parameter values may be efficiently derived without the need of non-linear optimisation techniques.

\section{REFERENCES}

[1] Casale, G., Harrison, P., and Vigliotti, M. Product-form approximation of tandem of queues via matrix geometric methods. In In Proc. of the 6th Int. Wor. on Numerical Solutions of Markov Chains (NSMC 2010) (Williamsburg, Virginia, 2010).

[2] Faugère, J. C., Gianni, P., Lazard, D., And MorA, T. Efficient computation of zero-dimensional Gröbner bases by change of ordering. J. Symb. Comput. 16, 4 (1993), 329-344.

[3] Harrison, P. G. Turning back time in Markovian process algebra. Theoretical Computer Science 290, 3 (January 2003), 1947-1986.

[4] Harrison, P. G., And Vigliotti, M. G. Response time distributions and network perturbation into product-form. In VALUETOOLS '09: Proc. of the

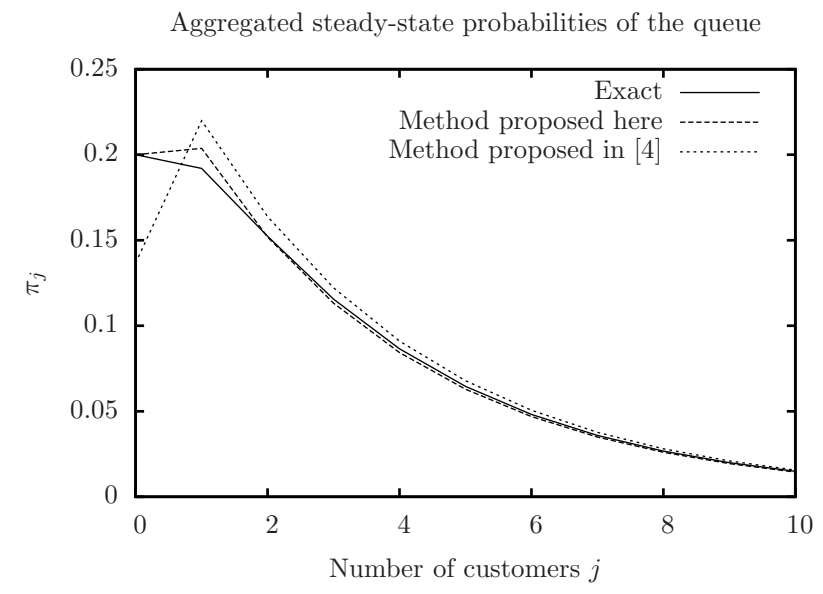

Figure 7: Comparison of aggregated steady-state probabilities of the $M / E_{2} / 1$ queue with its productform approximations. Parameters are $\lambda=0.8$ and $\mu=2$.

Fourth Int. ICST Conf. on Perf. Eval. Meth. and Tools (Pisa, Italy, 2009), ICST, pp. 1-9.

[5] Iverson, K. E. A programming language. John Wiley \& Sons, Inc., New York, USA, 1962.

[6] KAnT, K. Introduction to Computer System Performance Evaluation. McGraw-Hill, 1992.

[7] Kelly, F. Reversibility and stochastic networks. Wiley, New York, 1979.

[8] Kleinrock, L. Queueing Systems, vol. 1 (Theory). John Wiley and Sons, 1975.

[9] Latouche, G., and Ramaswami, V. Introduction to Matrix Anlytic Methods in Stochastic Modeling. Statistics and applied probability. ASA-SIAM, Philadelphia, PA, 1999.

[10] Latouche, G., and Ramaswami, Y. A logaritmic reduction algorithm for Quasi Birth and Death processes. J. of Appl. Prob. 30 (1994), 650-674.

[11] Marin, A., And Vigliotti, M. G. A general result for deriving product-form solutions of markovian models. In Proc. of First Joint WOSP/SIPEW Int. Conf. on Perf. Eng. (San Josè, CA, USA, 2010), ACM, pp. 165-176.

[12] Neuts, M. F. Matrix Geometric Solutions in Stochastic Models. John Hopkins, Baltimore, Md, 1981.

[13] Neuts, M. F. Explicit steady-state solutions to some elementary queueing models. Oper. Res. 30, 3 (1982), 480-489.

[14] Neuts, M. F. Structured stochastic matrices of $M / G / 1$ type and their application. Marcel Dekker, New York, 1989.

[15] Stewart, W. J. Probability, Markov Chains, Queues, and Simulation. Princeton University Press, UK, 2009.

\section{APPENDIX}

In this part we show an alternative proof of Thorem 1 that relies on the analysis of a $M / \mathrm{HYPO}_{K} / 1$ queue with homogeneous arrival process. 


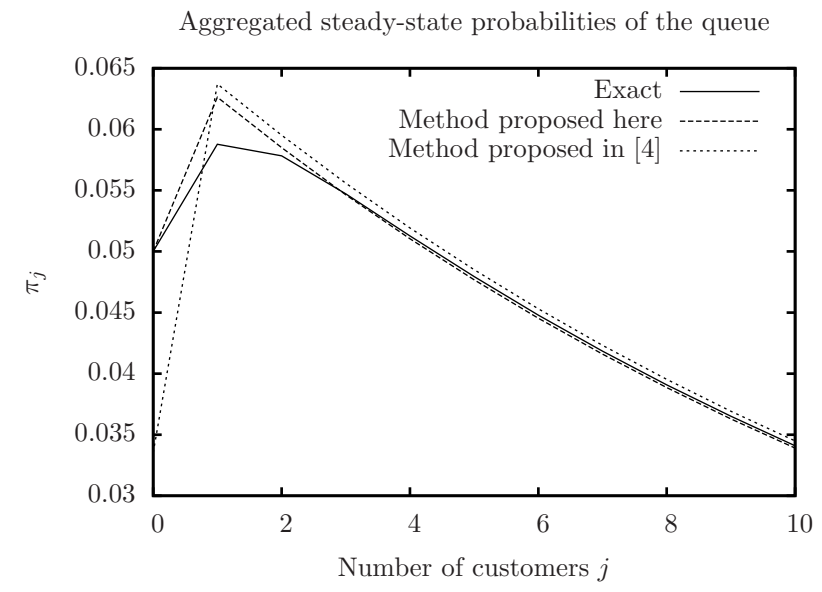

Figure 8: Comparison of aggregated steady-state probabilities of the $M / E_{2} / 1$ queue with its productform approximations. Parameters are $\lambda=0.95$ and $\mu=2$.

THEOREM 2. The steady-state probability distribution of a stable $M / \mathrm{HYPO}_{K} / 1$ queue is:

$$
\begin{cases}\pi_{0}=1-\sum_{k=1}^{K} \frac{\lambda}{\mu_{k}} & \\ \pi_{1 k}=\pi_{0} \frac{\lambda}{\mu_{k}} \prod_{z=k+1}^{K}\left(1+\frac{\lambda}{\mu_{z}}\right) & k=1, \ldots, K, \\ \boldsymbol{\pi}_{n+1}=\boldsymbol{\pi}_{n} \mathbf{R}, & n \geq 1\end{cases}
$$

where $\mathbf{R}$ is the matrix given by Definition 1.

Proof. The proof is structured as follows: first, we derive the vector of the un-normalised steady-state probabilities $\boldsymbol{\pi}^{\prime}$ assuming $\pi_{0}^{\prime}=1$ by solving the system of GBEs. Then, we compute the normalising constant.

Let us prove that matrix $\mathbf{R}$ is a solution of Equation (5). This implies that the GBEs for states $(n, k)$ with $n>1$ and $1 \leq k \leq K$ are satisfied. Since matrix $\mathbf{R}$ has an inverse by Lemma 1, Equation (5) can be conveniently rewritten as follows:

$$
\mathbf{R}^{-1} \mathbf{A}_{2}+\mathbf{A}_{1}+\mathbf{R} \mathbf{A}_{0}=\mathbf{0},
$$

where matrices $\mathbf{A}_{i}, i=0,1,2$, have been defined in Section 2.2. We observe that $\mathbf{R}^{-1} \mathbf{A}_{2}=\lambda \mathbf{R}^{-1}$ and that the elements of matrix $\mathbf{R} \mathbf{A}_{0}$ are:

$$
\left(\mathbf{R A} A_{0}\right)_{i j}=[j=1] r_{i n} \mu_{n}=[j=1] \lambda .
$$

Hence, element $z_{i j}$ of the matrix defined by the left-hand side of Equation (23) is:

$$
\begin{aligned}
z_{i j}= & \lambda\left(-[j=1]+[i=j]\left(1+\frac{\mu_{i}}{\lambda}\right)-[j=i+1] \frac{\mu_{i}}{\lambda}\right) \\
& -[i=j]\left(\lambda+\mu_{i}\right)+[j=i+1] \mu_{i}+[j=1] \lambda \\
= & -[j=1] \lambda+[i=j]\left(\lambda+\mu_{i}\right)-[j=i+1] \mu_{i} \\
& -[i=j]\left(\lambda+\mu_{i}\right)+[j=i+1] \mu_{i}+[j=1] \lambda=0 .
\end{aligned}
$$

The GBEs for states 0 and $(1, k)$ with $1 \leq k \leq K$ can be written as in Equation (3) and we assume $\pi_{0}^{\prime}=1$. It is easy to prove that:

$\left(\mathbf{A}_{1}+\mathbf{R} \mathbf{A}_{0}\right)_{i j}=[j=1] \lambda-[i=j]\left(\lambda+\mu_{i}\right)+[j=i+1] \mu_{i}$, which leads to the following system of equations:

$$
\left\{\begin{array}{l}
-\lambda+\pi_{1 K}^{\prime} \mu_{K}=0 \\
-\mu_{1} \pi_{11}^{\prime}+\lambda\left(\sum_{j=2}^{K} \pi_{1 j}^{\prime}\right)+\lambda=0 \\
\mu_{k-1} \pi_{1(k-1)}^{\prime}-\left(\lambda+\mu_{k}\right) \pi_{1 k}^{\prime}=0
\end{array} \quad 2 \leq k \leq K .\right.
$$

The first equation of System (24) straightforwardly gives $\pi_{1 K}^{\prime}=\lambda / \mu_{K}$. The last $K-1$ ones can be recursively solved obtaining the following expression for $\pi_{1 k}^{\prime}$ :

$$
\pi_{1 k}^{\prime}=\frac{\lambda}{\mu_{k}} \prod_{z=k+1}^{K}\left(1+\frac{\lambda}{\mu_{z}}\right) .
$$

The last step consists in showing that the expression for $\pi_{1 k}^{\prime}$ given by Equation (25) satisfies the second equation of System (24) that can be simplified to:

$$
1+\sum_{k=2}^{K} \frac{\lambda}{\mu_{k}} \prod_{z=k+1}^{K}\left(1+\frac{\lambda}{\mu_{z}}\right)=\prod_{z=2}^{K}\left(1+\frac{\lambda}{\mu_{z}}\right) .
$$

We proceed by induction on $K$. The base case, given for $K=1$, is trivial. Let us assume $K=t+1$, with $t \geq 1$. The left-hand side can be rewritten as:

$$
\begin{aligned}
1+\sum_{k=2}^{t} \frac{\lambda}{\mu_{k}} \prod_{z=k+1}^{t+1}\left(1+\frac{\lambda}{\mu_{z}}\right)+\frac{\lambda}{\mu_{t+1}} & =\left(1+\frac{\lambda}{\mu_{t+1}}\right)\left\{1+\sum_{k=2}^{t} \frac{\lambda}{\mu_{k}} \prod_{z=k+1}^{t}\left(1+\frac{\lambda}{\mu_{z}}\right)\right\} \\
= & \left(1+\frac{\lambda}{\mu_{t+1}}\right) \prod_{z=2}^{t}\left(1+\frac{\lambda}{\mu_{z}}\right)=\prod_{z=2}^{t+1}\left(1+\frac{\lambda}{\mu_{z}}\right),
\end{aligned}
$$

where the inductive hypothesis has been applied.

Note that as a consequence of Theorem 2 we know that matrix $\mathbf{R}$ has spectral radius strictly less than one (under stability assumption) and this implies that it is also the unique desired solution of matrix-equation (5) and, hence, Theorem 1 is proved. 